\title{
Clinical and histopathological principles for the diagnosis of a recurrent paraganglioma of the jugular foramen initially diagnosed as a middle ear adenoma: illustrative case
}

\author{
Jeffrey M. Breton, MD, ${ }^{1}$ Knarik Arkun, MD, ${ }^{1,2}$ Arthur S. Tischler, MD, ${ }^{2}$ Adnan S. Qamar, MBBS, MSc, ${ }^{2}$ Jonathan S. Sillman, MD, ${ }^{3}$ and \\ Carl B. Heilman, $\mathrm{MD}^{1}$

\begin{abstract}
${ }^{1}$ Department of Neurosurgery, Tufts Medical Center and Tufts University School of Medicine, Boston, Massachusetts; and Departments of ${ }^{2}$ Pathology and Laboratory Medicine
\end{abstract} \\ and ${ }^{3}$ Otolaryngology, Tufts Medical Center, Boston, Massachusetts
}

\begin{abstract}
BACKGROUND Paragangliomas (PGLs) are rare neoplasms that may be associated with hereditary PGL syndromes and variable risk of metastasis. Middle ear adenomas are extremely rare tumors with no known hereditary predisposition and extremely low risk of metastasis. Although often easily differentiated, they may share clinical and pathological features that misdirect and confuse the diagnosis.

OBSERVATIONS The authors discussed a 35-year-old woman with left-sided hearing loss and bleeding from the external ear canal who presented to an outside hospital. She underwent resection of a middle ear and mastoid mass, initially diagnosed as a middle ear adenoma with neuroendocrine features, with later mastoidectomy and ligation of the sigmoid sinus with microsurgical excision of persistent tumor in the jugular foramen and temporal bone. Histopathologically, her tumor was vascular, composed of benign-appearing epithelioid cells with "salt and pepper" neuroendocrine chromatin arranged in vague nests. Lesional cells were GATA3-immunopositive, glucagon-negative, and succinate dehydrogenase-immunonegative, consistent with PGL rather than middle ear adenoma, and required further workup for hereditary PGL syndromes.
\end{abstract}

LESSONS This case demonstrates potential challenges in differentiating a PGL from a middle ear adenoma. The authors offer clinical, histopathological, and imaging principles to aid in diagnosis and workup.

https://thejns.org/doi/abs/10.3171/CASE21307

KEYWORDS paraganglioma; middle ear adenoma; neuropathology; jugular foramen; skull base; middle ear neuroendocrine tumor; NET

Paragangliomas (PGLs) are usually benign neuroendocrine tumors (NETs) derived from paraganglion cells of the autonomic nervous system. Middle ear adenomas are rare, benign tumors with epithelial and neuroendocrine properties that originate from middle ear mucosal epithelium and often present with nonspecific findings on imaging. Here we describe an unusual jugular foramen and middle ear tumor initially presenting with hearing loss and bleeding from the left ear that was first concerning for middle ear adenoma with neuroendocrine features and later determined to be a GATA3positive and glucagon-negative PGL to illustrate clinical and pathological features of these two tumors.

\section{Illustrative Case}

A 35-year-old woman with history of migraine headaches presented to an outside hospital with left-sided hearing loss and bleeding from the left external ear canal. She did not have a personal history of hypertension, anxiety, palpitations, or syncope and denied a family history of pheochromocytoma or PGL. Imaging revealed a left mastoid and middle ear mass, which was partially resected during a left tympanoplasty and mastoidectomy. On pathology, her original tumor was diagnosed as a middle ear adenoma with neuroendocrine features possibly concerning for carcinoma. Five months later, she presented to our hospital with follow-up magnetic resonance imaging (MRI) that revealed persistent

ABBREVIATIONS CN = cranial nerve; $\mathrm{CT}=$ computed tomography; $I \mathrm{HC}=$ immunohistochemistry; MRI = magnetic resonance imaging; $\mathrm{NET}=$ neuroendocrine tumor; $\mathrm{PGL}$ = paraganglioma; $\mathrm{SDH}=$ succinate dehydrogenase.

INCLUDE WHEN CITING Published August 2, 2021; DOI: 10.3171/CASE21307.

SUBMITTED May 18, 2021. ACCEPTED May 20, 2021.

(C) 2021 The authors, CC BY-NC-ND 4.0 (http://creativecommons.org/licenses/by-nc-nd/4.0/). 

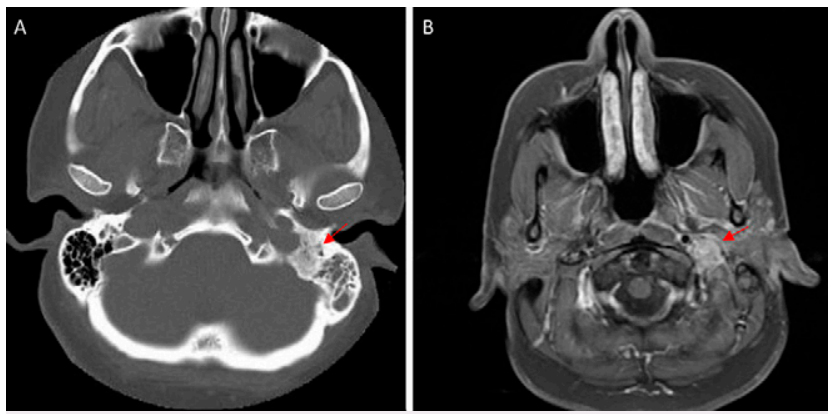

FIG. 1. A: Preoperative axial CT demonstrating a mass (red arrow) with associated bony erosion at the left jugular foramen and into mastoid air cells. B: Preoperative axial postcontrast T1-weighted MRI revealing a contrast-enhancing mass (red arrow) in the left jugular foramen with involvement of the petrous internal carotid artery.

tumor in the left jugular foramen extending down the jugular vein to the C1-2 level. She also complained of fatigue, persistent left hearing loss, tinnitus, difficulty with balance and coordination, frequent headaches, irregular heartbeat, and weight loss within the previous year. She denied dysphagia, hoarseness, or weakness lifting her shoulders.

On examination, her cranial nerves (CNs) were intact, with midline palatal elevation, full-strength bilateral sternocleidomastoid and trapezius muscles without atrophy, and tongue protruding midline and without atrophy. Her examination was otherwise unremarkable, with full strength and intact sensation in all extremities, normal reflexes, and absence of gait disturbance or dysmetria. T1-weighted MRI revealed a contrast-enhancing lesion of the left jugular foramen extending to C2 along the jugular vein and superiorly into the inferior aspect of the middle ear, with involvement of the petrous internal carotid artery (Fig. 1). Surgeons with neurosurgery and otolaryngology expertise performed a combined lateral skull base procedure, with mastoidectomy, left upper neck dissection, and ligation of the sigmoid sinus with microsurgical excision of the mass in the jugular foramen and temporal bone and placement of an abdominal fat graft. At the time of excision, the patient also received tympanoplasty. Postoperatively, the patient initially had a House-Brackmann grade III out of VI left facial palsy that subsequently resolved. She also developed a cerebrospinal fluid leak from the external auditory canal, which resolved with placement and subsequent removal of a lumbar drain.

On pathological examination, the gross tumor was a red-pink soft mass without hemorrhage. A frozen section was taken that was concerning for a PGL versus recurrent middle ear adenoma. Permanent section revealed a vascular tumor with focal nodular pattern and sclerosing pattern with extensive collagen deposition. Tumor cells were small to medium with fine, speckled chromatin and scanty cytoplasm. On immunohistochemistry (IHC), tumor cells stained positive for synaptophysin and were negative for multiple cytokeratin markers (pankeratin, CK5/6, CK7, CAM5.2) and p63. Mucicarmine stain and tyrosine hydroxylase immunostain also stained negative. Of note, tumor cells were positive for GATA3 with nuclear staining by IHC. IHC for SSTR2A revealed a granular cytoplasmic staining as opposed to an expected membranous staining pattern and was considered equivocal. Sustentacular cells stained immunopositive with S-100 and SOX-10. Staining for CD45 revealed rare inflammatory cells, and CD31 demonstrated rich vascularity. Ki-67 proliferative index was low ( 3\% to $5 \%)$. IHC staining for succinate dehydrogenase $(\mathrm{SDH}) \mathrm{B}$ was positive in endothelial cells, highlighting the vascular nature of the tumor and serving as an internal control, but was negative in tumor cells. Tumor cells were also negative for glucagon. This staining profile was consistent with an SDH-deficient PGL rather than a middle ear adenoma (Fig. 2). A local neck lymph node was resected and exhibited reactive features with no evidence of metastatic PGL.

The patient followed up with the endocrinology department, which recommended genetic testing for SDHB mutations in family members. She had normal prolactin level, which ruled out concurrent prolactinoma, and an unremarkable urinalysis upon screening for hematuria and renal cell carcinoma. She did not have elevated urine catecholamines or metanephrines when evaluated for associated pheochromocytoma. Genetics studies did not reveal a mutation in $S D H A, S D H B, S D H C, S D H D$, VHL, MEN1, NF1, RET, or VHL genes. Four months postoperatively, an octreotide scan showed residual uptake in the tumor bed, although temporal bone computed tomography (CT) and MRI of the internal auditory canal only revealed expected postsurgical change without evidence of persistent tumor. Although SSTR2A IHC was not interpretable in this case, positive SSTR2A can suggest that a tumor is amenable to ${ }^{68} \mathrm{Ga}$ Dotatate imaging ${ }^{1}$ and ${ }^{117}$ Lu-Dotatate therapy. ${ }^{2}$ Multidisciplinary discussions are ongoing to determine her optimal course for surveillance imaging in the future.

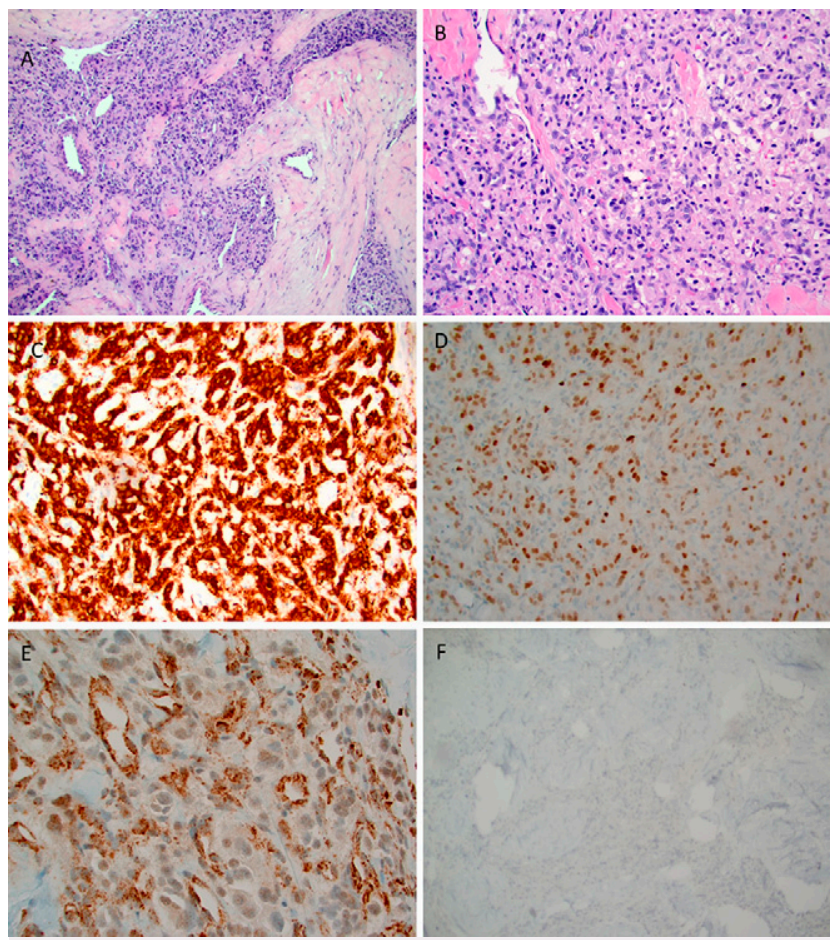

FIG. 2. A: Original magnification $\times 40$. Hematoxylin and eosin stain of the lesion showing moderately cellular lesion with sclerosing pattern. B: Original magnification $\times 400$. Hematoxylin and eosin stain showing vascular lesion with vague nodules and some nuclear atypia, without mitoses or necrosis. C: Original magnification $\times 400$. IHC for synaptophysin in lesional cells demonstrates strong cytoplasmic granular staining. D: Original magnification $\times 400$. On IHC for GATA3, nuclear immunostaining is present in lesional cells. E: Original magnification $\times 400$. IHC for SDHB. Note granular cytoplasmic staining in vessels with visible lumen and no staining in lesional cells of PGL. F: Original magnification $\times 400$. IHC for glucagon shows no staining in PGL cells. 


\section{Discussion \\ Observations}

In this case, the patient had a recurrent tumor involving the middle ear and jugular foramen that was initially diagnosed as an extremely rare tumor, a middle ear adenoma, and later correctly diagnosed as a rare, although more common, PGL. Although their typical clinical manifestations are often distinct, these tumors may also present with nonspecific findings on imaging, during surgery, and on histopathology. In these scenarios, correct diagnosis is vital because PGLs in this location have a strong association with germline mutations and should trigger a genetic workup. Because these tumors straddle a position between neurotology and skull base neurosurgery, it is valuable to delineate their clinical, surgical, and histopathological features to aid in future accurate diagnosis.

\section{Lessons}

PGLs are a genetically diverse set of paraganglion-derived NETs that exist on a spectrum of metastatic risk. The term glomus tumor is sometimes used by clinicians in referring to PGLs found in specific locations such as the jugular foramen (glomus jugulare) or the middle ear (glomus tympanicum), although there may be diagnostic overlap between them as well (jugulotympanic glomus tumor). Of note, middle ear PGLs make up approximately $30 \%$ to $40 \%$ of head and neck PGLs. In recent years, it has also become clearer that there are multiple hereditary PGL syndromes with associated genetic mutations in SDH in addition to well-known associations with VHL, NF1, and RET mutations. ${ }^{3}$ Diagnosis of PGL carries the highest genetic predisposition of any tumor, especially in head and neck locations, and warrants an extensive workup.

Classically, clinical suspicion for PGL or pheochromocytoma is based on symptoms of catecholamine excess, such as episodic headache, sweating, palpitations, or hypertension, although head and neck PGLs are usually nonsecretory, as in this case. The most common presenting symptoms for jugulotympanic PGLs are pulsatile tinnitus $(\sim 90 \%)$ and hearing loss ( $80 \%)$, although lower $C N$ deficits may also be seen. ${ }^{4}$ Suspicion may also be raised if patients present with associated diagnoses from known tumor syndromes such as von Hippel-Lindau or hereditary PGL syndromes or with family history of tumors or symptoms consistent with PGL or pheochromocytoma. Although these tumors are highly vascular and may bleed easily, it is uncommon for patients to present with bleeding from the ear because diagnosis is usually made before the tumor can erode through the soft tissues of the external ear canal. Traditional markers for diagnosis are plasma-free metanephrines and urinary fractionated metanephrines, ${ }^{5}$ although they are infrequently produced by head and neck PGLs, in which case plasma methoxytyramine levels may be more useful. ${ }^{6}$

Given their highly vascular nature, it is vital to image possible PGLs before attempting biopsy if they are grossly accessible in the external ear canal because bleeding may be difficult to control once it begins. These tumors tend to erode through neighboring structures and follow the path of least resistance, particularly through the temporal bone in the case of jugular PGL, which is easily visualized on CT, and into vessels, possibly invaginating into the lumen of jugular vein. They are highly contrast enhancing on both CT and $\mathrm{MRI}$, classically revealing a nonspecific "salt and pepper" appearance, although MRI is more sensitive and detects smaller PGLs. ${ }^{7}$ On diagnostic angiography, these tumors also reveal a clear vascular blush, which is absent in the case of middle ear adenoma. ${ }^{8}$
Treatment options include stereotactic radiosurgery and open surgery, frequently with preoperative embolization. Radiosurgery primarily controls growth of the tumor and may reduce symptoms as a primary or secondary modality. ${ }^{9,10}$ Resection is another treatment option, particularly if there is existing $\mathrm{CN}$ compression with associated neurological deficit. Embolization has been used preoperatively since the 1980 s to reduce intraoperative blood loss, ${ }^{11}$ although it is not curative by itself ${ }^{12}$ and may be associated with higher risk of postoperative $\mathrm{CN}$ deficits. ${ }^{13}$

Histopathology classically reveals a Zellballen pattern of nested cells with prominent vasculature and sustentacular cells circumscribing them. Sclerosing, pigmented, and clear patterns have also been recognized. Cells will often show "salt and pepper" nuclei, staining positive for neuroendocrine markers such as synaptophysin or chromogranin A and staining negative for cytokeratins. ${ }^{3}$ GATA3, a transcription factor binding to promoter GATA motifs that was initially recognized in breast duct and urothelial cells, is routinely used in $\mathrm{IHC}$ staining ${ }^{14}$ and is positive in up to $95 \%$ of cases of pheochromocytoma and $89 \%$ of cases of PGL, ${ }^{15}$ although it may be variable. Another key in the histopathological diagnosis of $\mathrm{PGL}$ is recognizing mutated SDH genes and their clinical implications. SDH is a multiunit enzyme in the Krebs cycle and electron transport chain that is vital for cellular respiration. The most common SDH mutation in familial head and neck PGL is in the SDHD subunit ( $66 \%)$, with SDHB being second most common $(\sim 15 \%)$, and then SDHC $(\sim 6 \%) .{ }^{16}$ Functional SDH activity may be seen with granular staining of SDHB in the cytoplasm of tumor cells, corresponding to the mitochondrial location of the enzyme complex. Absence of staining in tumor cells, with concomitant positive staining in nontumor tissue as an internal control, is suggestive of an SDH mutation. It does not reveal which specific SDH gene, however, because mutation in any subunit will alter the conformation of the entire complex and affect staining. Genetic subtyping is required for final diagnosis. SDHB-mutated PGL (PGL syndrome type 4) is the most concerning clinically because there is an elevated risk of metastasis $(23 \%$ versus low or $3 \%$ to $4 \%$ in other subtypes) and associated pheochromocytoma, renal cell carcinoma, gastrointestinal stromal tumor, and pituitary adenoma. ${ }^{3,17,18}$ There is also variable penetrance of this mutation, with approximately $21 \%$ penetrance by age 50 and $42 \%$ by age $70 ;{ }^{19}$ typically, the gene must be inherited from the father to develop tumors. ${ }^{20}$ The loss of tumoral SDHB immunoreactivity in this patient in the absence of a detected SDH gene mutation is currently unexplained but occasionally occurs epigenetically as a result of SDHC promoter methylation. ${ }^{21}$

First described in $1976,{ }^{22}$ middle ear adenomas are rare, benign tumors of the middle ear that classically present with unilateral hearing loss and have neuroendocrine and epithelial or glandular properties on pathology. This has led to multiple naming conventions and controversy regarding their cellular origin. Although they have recently been called middle ear adenoma with neuroendocrine features, they are more appropriately called middle ear NETs, in keeping with recent immunohistochemical findings and a World Health Organization initiative aimed to harmonize the nomenclature of NETs across disciplines and locations. ${ }^{23,24}$ Their preoperative diagnosis is difficult because they do not have specific imaging findings and are ideally diagnosed on histopathology. ${ }^{25}$ They have presented with facial nerve (CN VII) palsies, although that is rare. On T1weighted $\mathrm{MRI}$, they are isointense to hyperintense relative to white matter and gadolinium contrast enhancing. Although anatomically they 
TABLE 1. Characteristics of jugulotympanic paragangliomas and middle ear adenomas that may be useful in their diagnosis and workup

\begin{tabular}{|c|c|c|}
\hline & PGL & MEA \\
\hline Common presenting symptoms & Pulsatile tinnitus, unilateral hearing loss & $\begin{array}{l}\text { Unilateral hearing loss; may see nonpulsatile } \\
\text { tinnitus; } 25 \% \text { present w/ no symptoms }\end{array}$ \\
\hline $\begin{array}{l}\text { Associated w/ lower CN palsies } \\
\text { (especially CN X) }\end{array}$ & Yes & Uncommon; rare cases of CN VII palsy \\
\hline Contrast enhancing on $\mathrm{CT}$ or MRI & Yes & Yes, although variable \\
\hline Vascular blush on angiogram & Yes & No \\
\hline Glucagon IHC & Negative & May be strongly positive \\
\hline GATA3 IHC & Yes, $\sim 90 \%$ & Variable \\
\hline SDH mutations & Sometimes & No \\
\hline Resection as treatment & Yes, although SRS may be used & Yes, surgery is the mainstay of treatment \\
\hline Is SRS effective against it? & Yes & Typically avoided \\
\hline Is preop embolization useful? & Yes & Unlikely, although it has not been studied \\
\hline
\end{tabular}

MEA = middle ear adenoma; SRS $=$ stereotactic radiosurgery.

may be in proximity to Jacobson's nerve (tympanic branch of CN IX), they are detached and distinct on imaging, unlike the intimate involvement with Jacobson's nerve that may be seen with glomus tympanicum tumors. $^{8}$

On histopathology, middle ear adenomas are infiltrative and moderately cellular with variable architectures, including glandular/ tubular, trabecular, nested, cribriform, single cell, or cystic patterns and possibly form sheets. ${ }^{26,27}$ They are characterized by pancytokeratin, CAM5.2, EMA, chromogranin A, and synaptophysin immunoreactivity. With their epithelial and neuroendocrine lineage, they often show a dual staining pattern (e.g., CK7 staining luminal cells, p63 or CK5/6 staining abluminal cells). ${ }^{26,27}$ Of particular importance in this case, middle ear adenomas stain positive for glucagon ${ }^{26,28,29}$ and express other markers of hindgut NETs, which are derived from L-cells in the normal intestine. ${ }^{24}$ The significance of these cells in the middle ear is unknown. GATA3 immunostaining is usually negative or stains a small subset of luminal cells. ${ }^{24}$ Resection is the typical treatment of choice, with approach tailored to size and anatomy; transcanal tympanoplasty and radical mastoidectomy are frequently chosen. There is no clear role for somatostatin analogs, radiotherapy, or chemotherapy in their treatment, ${ }^{8}$ and given their fibrous and relatively avascular composition, ${ }^{30}$ preoperative embolization is unlikely to be effective, although it has not been studied.

These tumor types, although molecularly distinct and often clinically distinct, may be challenging to differentiate if they present with atypical clinical or anatomical features. By incorporating careful imaging and histopathological analyses pre-, intra-, and postoperatively, we diagnosed this unusual PGL that was initially mistaken for a middle ear adenoma. Table 1 demonstrates common features useful in distinguishing these skull base and middle ear tumors.

\section{References}

1. Chang CA, Pattison DA, Tothill RW, et al. (68)Ga-DOTATATE and (18)F-FDG PET/CT in paraganglioma and pheochromocytoma: utility, patterns and heterogeneity. Cancer Imaging. 2016; 16(1):22.

2. Kendi AT, Halfdanarson TR, Packard A, Dundar A, Subramaniam RM. Therapy with ${ }^{177}$ Lu-DOTATATE: clinical implementation and impact on care of patients with neuroendocrine tumors. AJR Am J Roentgenol. 2019;213(2):309-317.

3. Williams MD, Tischler AS. Update from the 4th edition of the World Health Organization Classification of Head and Neck Tumours: Paragangliomas. Head Neck Pathol. 2017;11(1):88-95.

4. Fayad JN, Keles B, Brackmann DE. Jugular foramen tumors: clinical characteristics and treatment outcomes. Otol Neurotol. 2010; 31(2):299-305.

5. Pacak K, Wimalawansa SJ. Pheochromocytoma and paraganglioma. Endocr Pract. 2015;21(4):406-412.

6. Rao D, Peitzsch M, Prejbisz A, et al. Plasma methoxytyramine: clinical utility with metanephrines for diagnosis of pheochromocytoma and paraganglioma. Eur J Endocrinol. 2017;177(2):103-113.

7. Vogl TJ, Bisdas S. Differential diagnosis of jugular foramen lesions. Skull Base. 2009;19(1):3-16.

8. Cardoso FA, Monteiro EMR, Lopes LB, Avila MNDC, Scarioli BO. Adenomatous tumors of the middle ear: a literature review. Int Arch Otorhinolaryngol. 2017;21(3):308-312.

9. Shapiro S, Kellermeyer B, Ramadan J, Jones G, Wiseman B, Cassis A. Outcomes of primary radiosurgery treatment of glomus jugulare tumors: systematic review with meta-analysis. Otol Neurotol. 2018;39(9):1079-1087.

10. Dobberpuhl MR, Maxwell S, Feddock J, St Clair W, Bush ML. treatment outcomes for single modality management of glomus jugulare tumors with stereotactic radiosurgery. Otol Neurotol. 2016;37(9): $1406-1410$ 
11. Murphy TP, Brackmann DE. Effects of preoperative embolization on glomus jugulare tumors. Laryngoscope. 1989;99(12):1244-1247.

12. Tasar M, Yetiser S. Glomus tumors: therapeutic role of selective embolization. J Craniofac Surg. 2004;15(3):497-505.

13. Gaynor BG, Elhammady MS, Jethanamest D, Angeli SI, Aziz-Sultan MA. Incidence of cranial nerve palsy after preoperative embolization of glomus jugulare tumors using Onyx. J Neurosurg. 2014;120(2): 377-381.

14. Zheng R, Blobel GA. GATA transcription factors and cancer. Genes Cancer. 2010;1(12):1178-1188.

15. Nonaka D, Wang BY, Edmondson D, Beckett E, Sun C-CJ. A study of gata3 and phox $2 b$ expression in tumors of the autonomic nervous system. Am J Surg Pathol. 2013;37(8):1236-1241.

16. Pasini $B$, Stratakis $C A$. SDH mutations in tumorigenesis and inherited endocrine tumours: lesson from the phaeochromocytoma-paraganglioma syndromes. J Intern Med. 2009;266(1):19-42.

17. Boedeker CC, Hensen EF, Neumann HPH, et al. Genetics of hereditary head and neck paragangliomas. Head Neck. 2014;36(6): 907-916.

18. van Hulsteijn LT, Dekkers OM, Hes FJ, Smit JWA, Corssmit EPM. Risk of malignant paraganglioma in SDHB-mutation and SDHDmutation carriers: a systematic review and meta-analysis. J Med Genet. 2012;49(12):768-776.

19. Rijken JA, Niemeijer ND, Jonker MA, et al. The penetrance of paraganglioma and pheochromocytoma in SDHB germline mutation carriers. Clin Genet. 2018;93(1):60-66.

20. Baysal BE, Maher ER. 15 years of paraganglioma: genetics and mechanism of pheochromocytoma-paraganglioma syndromes characterized by germline SDHB and SDHD mutations. Endocr Relat Cancer. 2015;22(4):T71-T82.

21. Bernardo-Castiñeira C, Valdés N, Sierra MI, et al. SDHC promoter methylation, a novel pathogenic mechanism in parasympathetic paragangliomas. J Clin Endocrinol Metab. 2018;103(1):295-305.

22. Hyams VJ, Michaels L. Benign adenomatous neoplasm (adenoma) of the middle ear. Clin Otolaryngol Allied Sci. 1976;1(1):17-26.

23. Rindi G, Klimstra DS, Abedi-Ardekani B, et al. A common classification framework for neuroendocrine neoplasms: an International Agency for Research on Cancer (IARC) and World Health Organization (WHO) expert consensus proposal. Mod Pathol. 2018;31(12): 1770-1786.
24. Asa SL, Arkun K, Tischler AS, et al. Middle ear "adenoma": a neuroendocrine tumor with predominant $\mathrm{L}$ cell differentiation. Endocr Pathol. Published online May 27, 2021. doi:10.1007/s12022-02109684-z34041698

25. Zan E, Limb CJ, Koehler JF, Yousem DM. Middle ear adenoma: a challenging diagnosis. AJNR Am J Neuroradiol. 2009;30(8): 1602-1603.

26. Torske KR, Thompson LDR. Adenoma versus carcinoid tumor of the middle ear: a study of 48 cases and review of the literature. Mod Pathol. 2002;15(5):543-555.

27. Katabi N. Neuroendocrine neoplasms of the ear. Head Neck Pathol. 2018;12(3):362-366.

28. Bell D. Ear: external, middle, and temporal bone. In: Gnepp's Diagnostic Surgical Pathology of the Head and Neck. 3rd ed. Elsevier; 2021:927-972.

29. Ketabchi S, Massi D, Franchi A, Vannucchi P, Santucci M. Middle ear adenoma is an amphicrine tumor: why call it adenoma? Ultrastruct Pathol. 2001;25(1):73-78.

30. Bierry G, Riehm S, Marcellin L, Stierlé J-L, Veillon F. Middle ear adenomatous tumor: a not so rare glomus tympanicum-mimicking lesion. J Neuroradiol. 2010;37(2):116-121.

\section{Disclosures}

The authors report no conflict of interest concerning the materials or methods used in this study or the findings specified in this paper.

\section{Author Contributions}

Conception and design: Breton, Arkun, Sillman, Heilman. Acquisition of data: all authors. Analysis and interpretation of data: Breton, Arkun, Qamar, Heilman. Drafting the article: Breton, Arkun, Heilman. Critically revising the article: Breton, Arkun, Tischler, Sillman, Heilman. Reviewed submitted version of manuscript: Breton, Arkun, Tischler, Sillman, Heilman. Approved the final version of the manuscript on behalf of all authors: Breton. Study supervision: Arkun.

\section{Correspondence}

Jeffrey M. Breton: Tufts Medical Center, Boston, MA. jeffrey.breton@ tufts.edu. 